\title{
Suicide gene therapy in head and neck carcinoma cells: An in vitro study
}

\author{
MARIANNE SCHMIDT, PETRA GRUENSFELDER, JEANETTE ROLLER and RUDOLF HAGEN
}

University of Wuerzburg, Department of Otorhinolaryngology, Josef-Schneider-Strasse 11, D-97080 Würzburg, Germany

Received October 18,2010; Accepted December 20, 2010

DOI: 10.3892/ijmm.2011.610

\begin{abstract}
The aim of gene therapy includes the tight spatial and temporal control of transgenic expression. There are several approaches concerning the externally inducible gene promoters used for the control of suicide genes. We have tested the mifepristone-dependent system GeneSwitch to regulate the expression of a deletion mutant of Pseudomonas exotoxin $\mathrm{A}$ in the hypopharyngeal carcinoma cell line, FADU. The GeneSwitch system consists of two plasmids, the regulatory plasmid, pSwitch, and the pGene/V5-His plasmid, in which we cloned the toxin mutant (pGene/V5-His-ETA). We stably transfected FADU cells with pSwitch and subsequently transiently separated pSwitch clones with pGene/ V5-His-ETA. We tested the inductive capacities of single pSwitch clones, the influence of experimental variations in transfection, the inductive capacities without antibiotic selection pressure, the inductive capacity after re-induction, as well as the background expression levels. In FADU cells the GeneSwitch-ETA combination worked precisely and effectively. Our in vitro study revealed that the use of toxin genes in combination with the GeneSwitch system is a promising approach for gene therapy in head and neck cancer.
\end{abstract}

\section{Introduction}

During recent years, different basic approaches for gene therapy in head and neck cancer treatment have been investigated. A general aim of gene therapy strategies is the spatial and temporal defined expression of suicide genes, such as thymidine kinase or bacterial toxins. A temporally defined switch of gene expression can be achieved with gene promoters, which are controlled by exogenous stimuli like hormones, gamma irradiation or hyperthermia. In a former study we tested two environmentally inducible promoter

Correspondence to: Dr Marianne Schmidt, Department of Otorhinolaryngology, University of Wuerzburg, Josef-SchneiderStrasse 11, D-97080 Würzburg, Germany

E-mail: schmidt_m2@klinik.uni-wuerzburg.de

Key words: head and neck cancer, suicide gene therapy, Pseudomonas exotoxin A, GeneSwitch systems in head and neck cancer cell lines: the heat-sensitive heat shock protein (Hsp70) promoter and a synthetic construct using radiation-inducible enhancer elements of the early growth response 1 (Egr1) gene promoter (1). Results of that study were disappointing. Reporter gene induction was low and background expression without external stimuli proved to be too high for the usage of suicide genes. For this reason we tested the GeneSwitch ${ }^{\mathrm{TM}}$ system for induction of the reporter gene EGFP as well as a construct containing a deleted version of Pseudomonas exotoxin A. The GeneSwitch system is a mifepristone-inducible mammalian expression system originally developed by Wang et al (2) that uses a combination of regulatory components derived from various human and yeast transcription factors. It is based on a mutated human progesterone receptor which is unable to bind endogenous hormone but is activated by binding of the progesterone antagonist, mifepristone (RU-486) $(2,3)$.

The chimeric transactivator (regulator) protein of the GeneSwitch system comprises the GAL4 DNA-binding domain from Saccharomyces cerevisiae fused to the ligandbinding domain of a mutant progesterone receptor and the activation domain of the p65 subunit of human NF- $\kappa B$ (3). In the presence of mifepristone, the regulator protein binds to GAL4 activation sequences of the inducible transgene, inducing transcription of the target gene (4). Although mifepristone has antiprogesterone activity, the concentration needed for gene induction is much lower than the concentration producing an anti-progesterone effect in humans (5). The GeneSwitch system comprises the mifepristone-inducible pSwitch vector, as well as the pGene vector for cloning of the target gene. We generated a deletion mutant of Pseudomonas exotoxin A (ETA) cDNA, lacking the binding domain of the toxin and cloned this construct into the pGene vector version A. Furthermore, we cloned EGFP as a reporter gene into the pGeneA vector.

We generated stable pSwitch clones of the head and neck squamous carcinoma cell line, FADU, and subsequently transiently transfected these clones with the pGene vector containing either EGFP or the deletion mutant of ETA. We tested the inductive capacities of single pSwitch clones, the influence of different DNA amounts of linearized or circular DNA used for transfection, the inductive capacities without antibiotic selection pressure, the inductive capacity after mifepristone treatment in surviving cells, as well as the background expression levels. 
The results indicate, that the GeneSwitch system-ETA combination might be a suitable approach for suicide gene therapy in human head and neck cancer.

\section{Materials and methods}

Cell lines and cell culture. The head and neck squamous carcinoma cell line FADU (American Type Culture Collection, atcc.org) had been established from a pharyngeal squamous cell carcinoma. The cell line was grown with RPMI-1640 medium (Seromed, Munich, Germany), supplemented with $10 \%$ fetal calf serum (FCS).

Construction of pGene/V5-His-ETA. Pseudomonas aeruginosa exotoxin A cDNA in pUC19 was kindly provided by Dr Winfried Wels, Georg-Speyer-Haus, Institute for Biomedical Research, Frankfurt/Main (Germany). The GeneSwitch ${ }^{\mathrm{TM}}$ core kit was purchased from Invitrogen (Karlsruhe, Germany). The protein domains of ETA are graphically described in Fig. 1. We deleted the binding domain of ETA by performing a PCR, spanning the translocation and ADP-ribosylation domains. With this PCR we further inserted a Kozak-sequence for the intracellular translation as well as a HindIII site at the $5^{\prime}$ and an EcoRI site at the 3 ' end (Fig. 2).

PCR was carried out with $0.5 \mathrm{ng}$ pUC19-ETA, $10 \mathrm{pmol}$ of each primer, 1 Unit AmpliTaq Gold DNA Polymerase (Applied Biosystems, Darmstadt, Germany) at the following conditions: 2 min denaturation at $94^{\circ} \mathrm{C}$ followed by 37 cycles with $94^{\circ} \mathrm{C}$ for $45 \mathrm{sec}$ denaturation, $30 \mathrm{sec}$ annealing at $50^{\circ} \mathrm{C}$ and $2 \mathrm{~min}$ elongation at $72^{\circ} \mathrm{C}$. PCR was completed by a final extension at $72^{\circ} \mathrm{C}$ for $15 \mathrm{~min}$. The PCR primers were ETA-forward, 5'-GATCAAGCTTCCACATGG CCGAGCAACGCTGCAACCTCGAC-3'; ETA-reverse, 5'-GATCGAATTCATATTCGATTGGGCTGGCATC-3'. The PCR product was purified from a $1 \%$ agarose gel and subsequently digested with EcoRI and HindIII. The pGene/ V5-HisA plasmid likewise was cut with EcoRI/HindIII and the purified PCR-product was ligated into the digested and purified vector. Both the $5^{\prime}$ and $3^{\prime}$ ends of the cloned insert were sequenced. In order to avoid mutations caused by PCR a $B g l \mathrm{II} / E c o$ RI fragment of the PCR-insert, spanning the ETA domains II, Ib and III was in the end replaced by the original $B g l \mathrm{II} / E c o$ RI fragment of pUC19-ETA.

Construction of pGene/V5-His-EGFP. Both pGene/V5-HisA (Invitrogen, Darmstadt, Germany) and pEGFP-N1 (BectonDickinson, Heidelberg, Germany) were digested with BamHI and NotI. The EGFP-insert was purified from an agarose gel and ligated with the BamHI/NotI restricted and gel-purified pGene/V5-HisA vector DNA.

DNA clean-up and preparation. All preparations were performed with kits from Qiagen (Hilden, Germany) according to the kit manuals.

Transfection with $p$ Switch/pGeneV5-His. Prior to transfection pSwitch was linearized with Bst 11071 . Both linearized and circular pSwitch were used for transfections. pGene/ V5-His-ETA was linearized with $N s b$ I. pGene/V5-His-EGFP was digested with $N s b I$. Linearized plasmids were gel-purified.

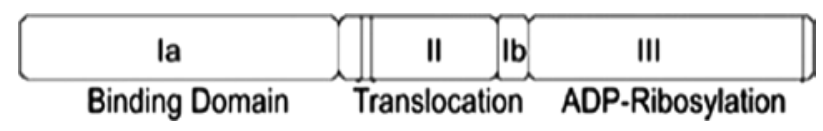

Figure 1. Protein domains of Pseudomonas exotoxin A (ETA).

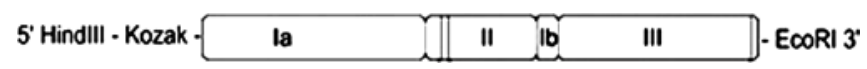

Figure 2. PCR construct.

For transfection of FADU cells the MATra (Magnet Assisted Transfection) system was used (IBA BioTAGnology, Goettingen, Germany) (6). Transfections were performed according to the manufacturer's protocol. Briefly, FADU cells were diluted to $5 \times 10^{5}-1 \times 10^{6}$ cells $/ \mathrm{ml}$. The cell suspension was mixed with $30 \mu \mathrm{l} / \mathrm{ml}$ MATra-S in 6-well plates. At the same time 3, 6, 9 or $12 \mu \mathrm{g}$ DNA were diluted with serum-free RPMI medium to an end volume of $200 \mu \mathrm{l}$. Subsequently to each of these DNA dilutions, $3 \mu$ l MATra-A reagent $/ \mu \mathrm{g}$ DNA was added. The preparations were mixed and incubated for $20 \mathrm{~min}$. During incubation time the medium in the 6-well plates was replaced by $2 \mathrm{ml}$ serum-free RPMI medium. After $20 \mathrm{~min}, 200 \mu \mathrm{l}$ of the bead-DNA-mixture were added to each well, mixed and placed on a universal magnet plate (IBA BioTAGnology) for $15 \mathrm{~min}$. After magnetic incubation the serum-free medium was replaced by normal culture medium.

For stable transfections with pSwitch, cells received $400 \mu \mathrm{g} / \mathrm{ml}$ hygromycin $48 \mathrm{~h}$ after transfection. Single pSwitchClones were isolated using the ring technique (7). We further tried to establish stable double transfected cell clones with pSwitch and pGene/V5-His-ETA by double selection with hygromycin and zeozin. However, treatment with both antibiotics caused cell death after a few days in culture. For this reason we transfected the stable pSwitch clones transiently with the target gene construct.

Gene induction. For gene induction, $1 \times 10^{-8} \mathrm{M}$ mifepristone were added to the double transfected cells $48 \mathrm{~h}$ after transient transfection with pGene/V5-His-ETA or pGene/V5-His-EGFP, respectively. Cells were tested for target gene expression after different incubation times post-induction (1, 2, 3, 4 days). Furthermore, we tested the stability of $\mathrm{pSwitch} / \mathrm{pGene} /$ V5-HisA-ETA double transfections over longer time periods without antibiotic selection pressure.

Fluorescence microscopy. EGFP-expression was examined microscopically with a Leica inverse fluorescence microscope (Leica, Wetzlar, Germany).

Cell viability and proliferation assay. Double transfected FADU cells were seeded at 3000 cells/well in 96-well plates with RPMI, supplemented with $400 \mu \mathrm{g} / \mathrm{ml}$ hygromycin. After $24 \mathrm{~h}$ cells were induced with $1 \times 10^{-8} \mathrm{M}$ mifepristone. Cell viability after induction of the ETA-gene was measured by replacing the culture medium with medium containing MTT $(1 \mathrm{mg} / \mathrm{ml})$. After $4 \mathrm{~h}$ incubation, MTT-staining solution was replaced by isopropanol and cells were incubated at $37^{\circ} \mathrm{C}$ overnight. The colour conversion of MTT to a blue formazan 


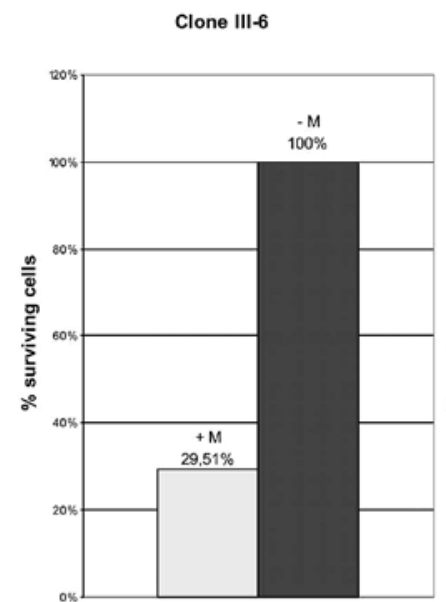

clone $1-4$

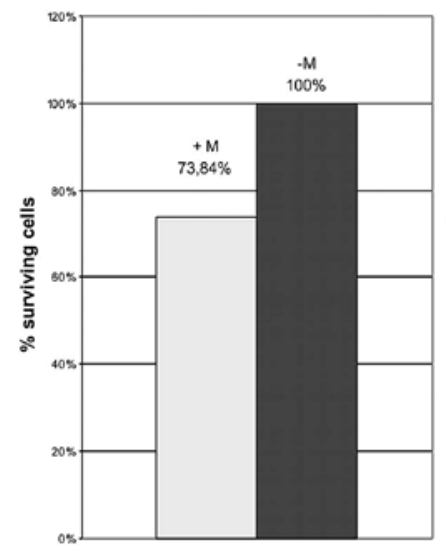

Clone V-5

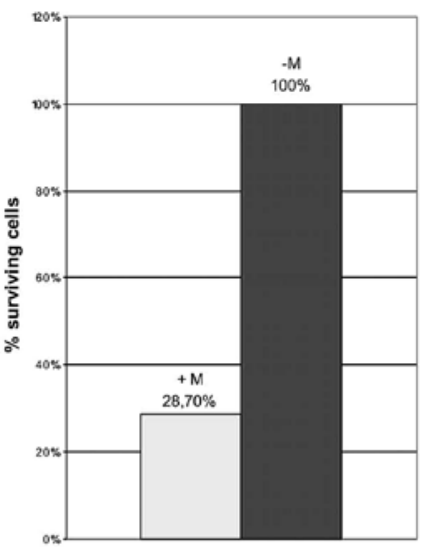

Clone II-4

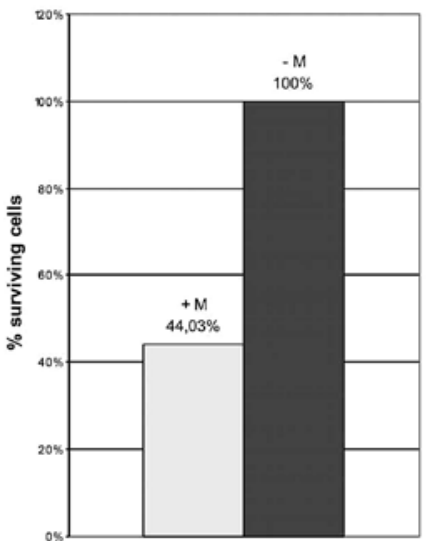

Clone III-9

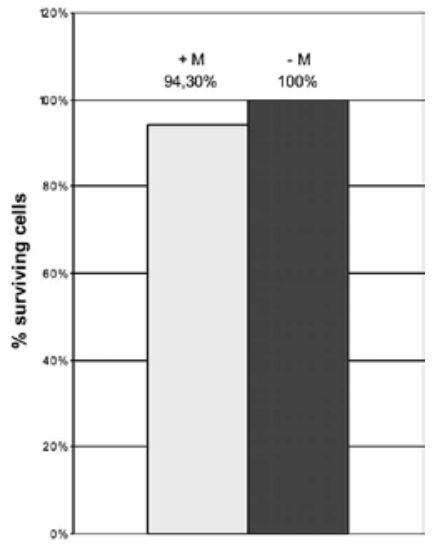

Clone III-5

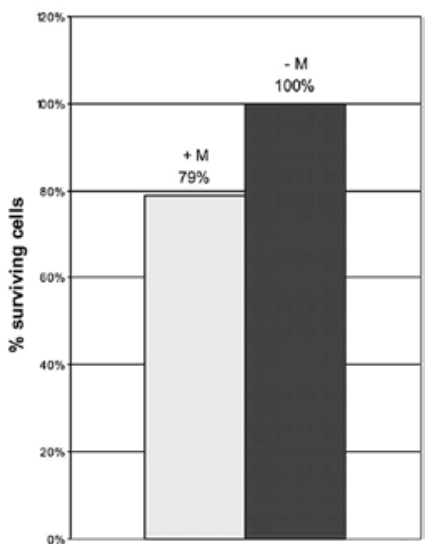

Figure 3. Inductive capacity of different stable $\mathrm{pS}$ witch clones after transfection with pGeneA-ETA (3 $\mu \mathrm{g}$ DNA) and induction with mifepristone (+M). Transiently transfected but uninduced control cells are denoted as -M.

Clone II-4

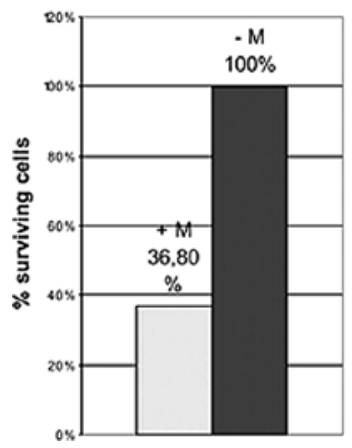

Clone II-4

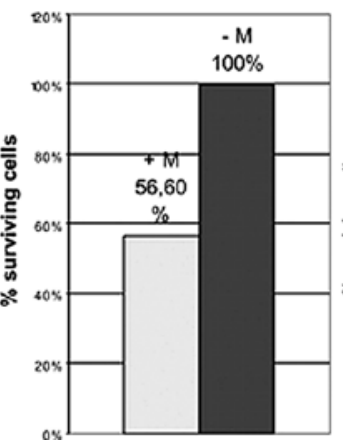

Clone II-4

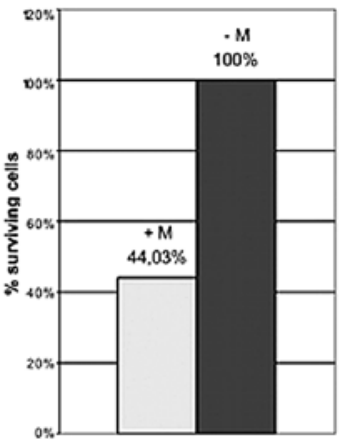

Clone II-4

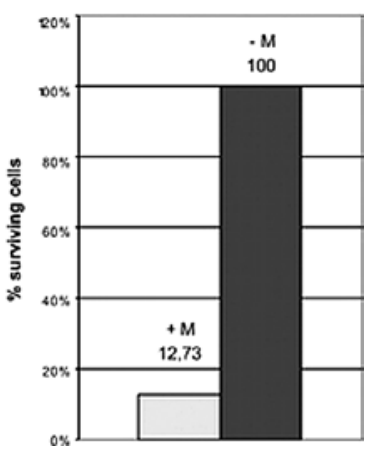

Figure 4. pSwitch clone II-4 was transiently transfected with $3 \mu \mathrm{g}$ pGeneA-ETA in four independent experiments under the same experimental conditions.

dye was measured with an ELISA reader at a wavelength of $570 \mathrm{~nm}$. The amount of the formazan dye is in direct proportion to the number of metabolically active cells in the culture.

For re-induction experiments we used the Cell Counting kit-8 (Dojindo Laboratories, Kumamoto, Japan), which utilizes a water-soluble tetrazolium salt. This assay allows measurement of cell viability several times successively and was performed as described in the manufacturer's manual.

\section{Results}

Inductive capacities of stable p Switch clones. Inductivity of the stable pSwitch clones transfected with pGeneA-ETA varied strongly between single clones and from transfection to transfection. Representative examples of 6 different pSwitch clones, each transfected with $3 \mu \mathrm{g}$ pGeneA-ETA DNA are shown in Fig. 3. We picked out clone II-4 and tested this clone 
Clone II-4, linearized pGene. ETA

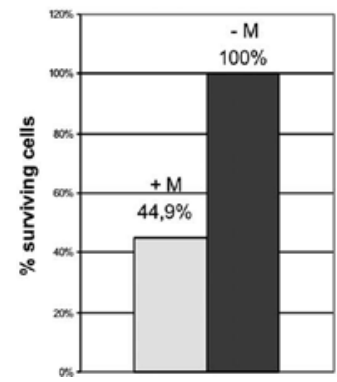

Clone II-4, linearized pGeneETA

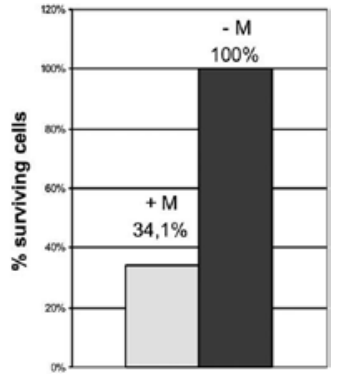

Figure 5. pSwitch clone II-4 was transiently transfected with $3 \mu \mathrm{g} \mathrm{NsbI-}$ linearized and gel-purified pGeneA-ETA in two independent experiments under the same experimental conditions. in several transient transfections, each time with $3 \mu \mathrm{g}$ DNA and under the same experimental conditions. The results are summarized in Fig. 4.

The variation of the expression rates can be explained by the integration of plasmid DNA at different chromosomal sites. The cell cycle phase of the transfected celsl might also play a role.

Inductive capacities under differential experimental conditions. We first tested if transfection with linearized plasmids can raise efficacy of gene induction. The cell survival after transient transfection of clone II-4 with $3 \mu \mathrm{g}$ pGeneA-ETA DNA, which was linearized with $N s b \mathrm{I}$, and subsequently gel purified is illustrated in Fig. 5. Variations in the expression
Clone II-4, $3 \mu g$ DNA

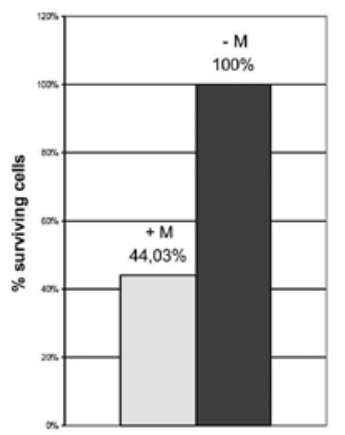

Clone $\|-4,6 \mu g$ DNA

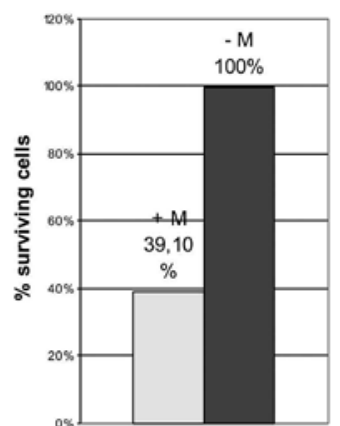

Clone $11-4,9 \mu g$ DNA

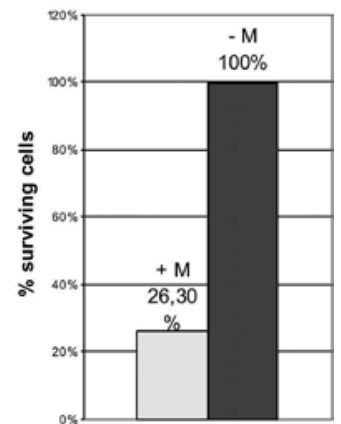

Clone II-4, $12 \mu g$ DNA

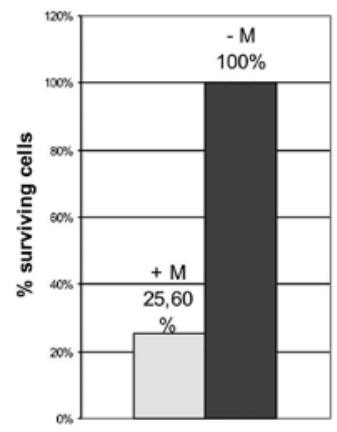

Clone II-4, $15 \mu \mathrm{g}$ DNA

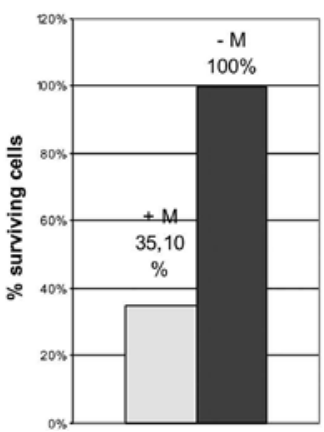

Figure 6. pSwitch clone II-4 was transiently transfected with 3, 6, 9, 12 or $15 \mu \mathrm{g}$ circular pGeneA-ETA plasmid.

$24 \mathrm{~h}$ post-induction

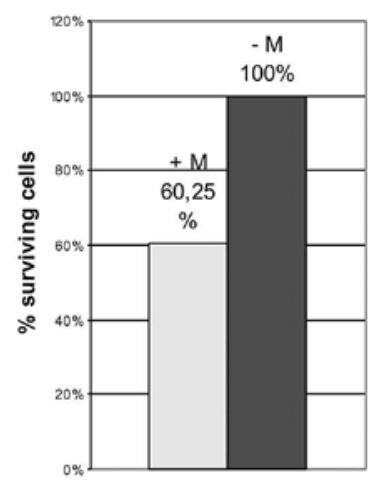

$48 \mathrm{~h}$ post-induction

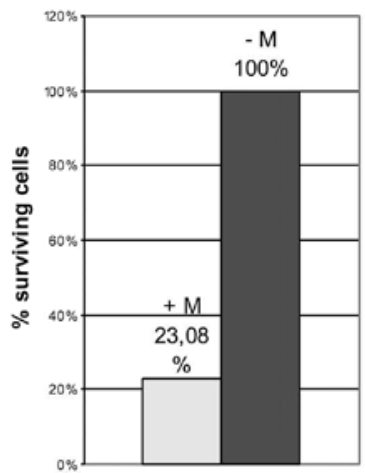

$72 \mathrm{~h}$ post-induction

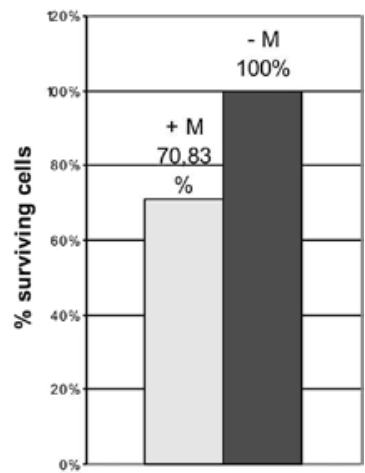

Figure 7. pSwitch clone II-4 was transiently transfected with $12 \mu \mathrm{g}$ circular pGeneA-ETA plasmid. Survival of cells was measured 24,48 or $72 \mathrm{~h}$ after induction with mifepristone. 


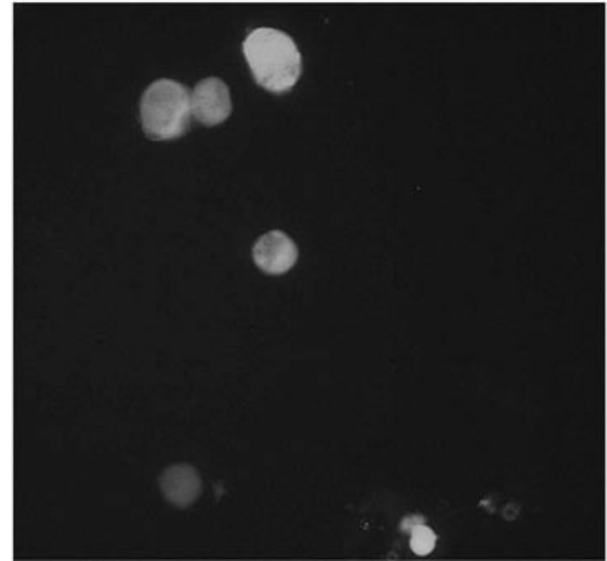

Figure 8. Induced expression of EGFP in transfected cells.

rates were similar as in the experiments with circular DNA. Furthermore, we were not able to observe a striking benefit when plasmid DNA was linearized prior to transfection.

As a further experimental variation we increased DNA amounts of pGene/V5-His-ETA for the transient transfections. As displayed in Fig. 6, increasing DNA amounts clearly enhanced gene expression up to an amount of $12 \mu \mathrm{g}$ DNA per transient transfection.

In order to determine the point of time, at which the effect of gene activation is most obvious we measured cell survival at different stages after mifepristone application. We transfected FADU cells of clone II-4 transiently with pGene/ V5-His-ETA, induced gene expression after $24 \mathrm{~h}$ with mifepristone and measured cell death after further 24,48 or $72 \mathrm{~h}$. The results are summarized in Fig. 7. The strongest effect of gene induction was reached after $48 \mathrm{~h}$. It is likely that $72 \mathrm{~h}$ after hormone-induction surviving cells have already begun proliferation, resulting in blurring of the suicide effect.

Induced expression of reporter gene EGFP. In order to visualize the gene expression we transfected stable pSwitch clones with EGFP as a reporter gene. Qualitative analysis revealed a lower inductivity, compared to transfection with ETA (Fig. 8). Probably only a few molecules of ETA are sufficient for cell killing, while higher amounts of EGFP are necessary for visualisation.

Inductive capacity after longer time periods without antibiotic selection pressure. An interesting question was how long the double transfected cells can be induced to express the toxic protein, when they are grown without antibiotic selection pressure. This question is especially interesting with regard to the use of the GeneSwitch system in vivo. We transiently transfected pSwitch clone II-4 with pGene/V5-His-ETA and tested gene induction of ETA after 2 or 42 days. Results are displayed in Fig. 9. After 2 days with or without antibiotic selection there was no striking difference in inductivity between the cells kept with or without hygromycin. After 42 days however, cells grown without hygromycin were less inducible. Nevertheless, there was still a cell killing rate of over $50 \%$, which makes the system attractive for further experiments.

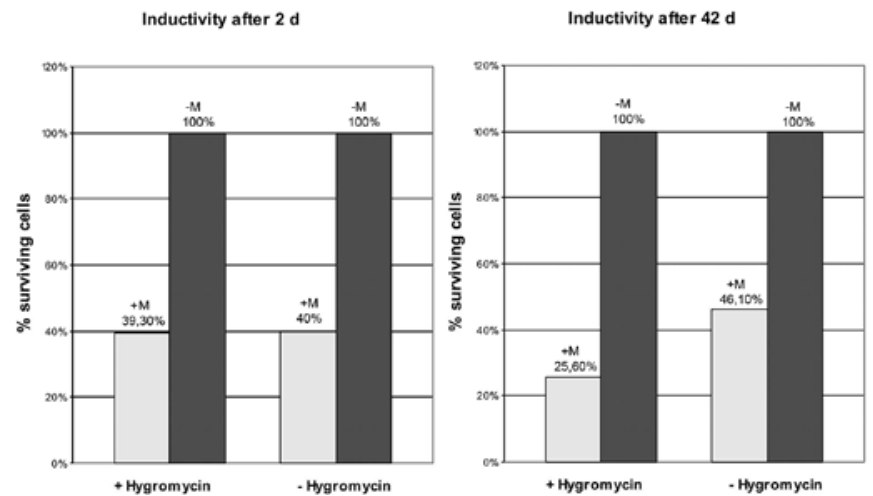

Figure 9. pSwitch clone II-4 was transiently transfected with $12 \mu \mathrm{g}$ circular pGeneA-ETA plasmid after 2 or 42 days and subsequently induced with mifepristone $(+\mathrm{M},-\mathrm{M})$. One cell fraction was kept with antibiotic selection pressure, one without.

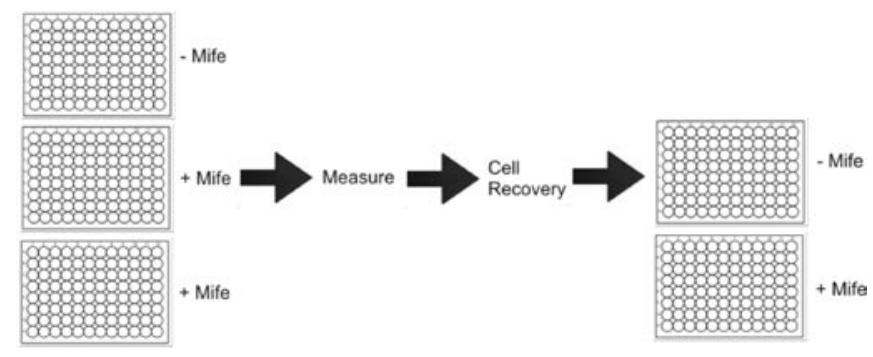

Figure 10. Schematic procedure of the re-induction experiment.

Re-induction after a recovery period. An interesting question concerned the fraction of surviving cells. Are these cells uninducible or is there a temporary lack of inducibility? We performed cytotoxicity testing with the Cell Counting kit-8, which allows the measurement of the same cell population several times. First we compared cells induced with mifepristone and uninduced cells. After the first induction we allowed the cells to grow until the population had recovered (4 days). We then, induced a second time and compared the cells with uninduced cells of the same population (schematic depiction of experimental design in Fig. 10).

As shown in Fig. 11 the first induction with mifepristone resulted in a cell killing rate of about $60 \%$. The surviving fraction proved to be much less inducible. Only $20 \%$ of the remaining cell fraction died after induction of pGeneA-ETA. Presumably the main fraction of surviving cells is marked by unfavourable integration of the ETA gene, resulting in a silenced transgene.

Background expression. To test the possible background expression of the target gene, we compared cells transfected solely with pSwitch with cells transfected with both plasmids with and without mifepristone induction. Fig. 12 summarizes the results of this experiment.

Cells transfected with pSwitch and pGene-ETA but not induced showed a somewhat lower survival rate than cells transfected exclusively with pSwitch, which indicates, that the GeneSwitch system is not completely restrictive. However, the background expression was very low compared to other inducible gene reporter systems. 
Induction 1

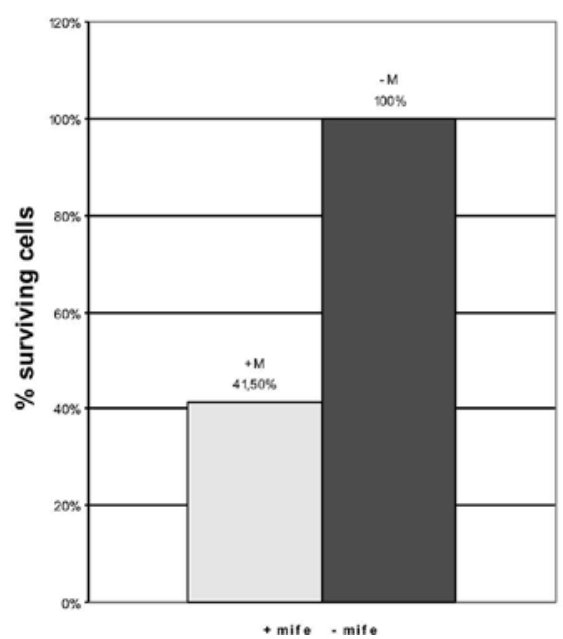

Induction 2

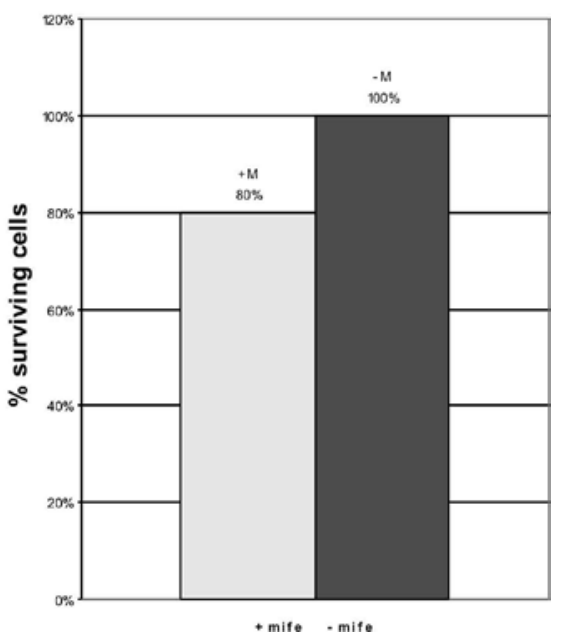

Figure 11. pSwitch clone II-4 was transiently transfected with $4 \mu \mathrm{g}$ circular pGeneA-ETA plasmid and subsequently induced with mifepristone (+M, -M). According to the protocol displayed in Fig. 10 surviving cells of the first induction were induced again after a short recovery period of 4 days.

Background expression

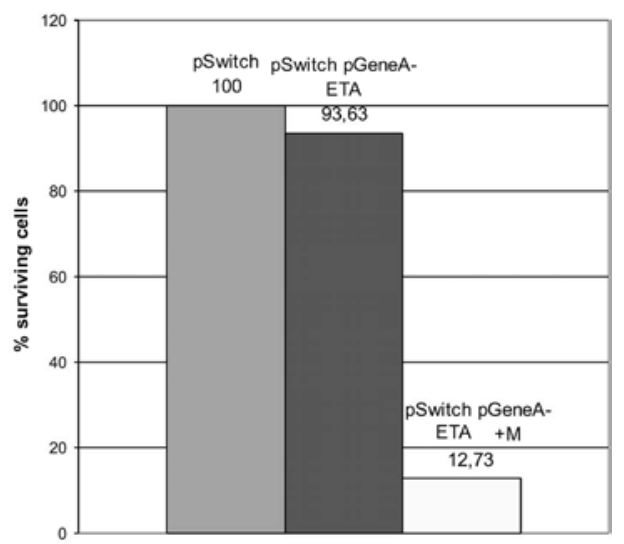

Figure 12. Comparison of FADU cells transfected with pSwitch only, pSwitch and pGene-ETA, either uninduced $(-\mathrm{M})$ or induced with mifepristone $(+\mathrm{M})$.

\section{Discussion}

Bacterial toxins have been extensively tested as therapeutic agents in cancer therapy. Pseudomonas exotoxin, a $66.5 \mathrm{kDa}$ protein consisting of 3 domains is among the most potent of the naturally occurring bacterial toxins. Its domain Ia mediates binding to the cell surface, domain II catalyzes the translocation into the cytoplasm, and domain II contains the ADP ribosylation activity. ADP ribosylation inhibits protein synthesis by inactivation of the elongation factor-2 (EF-2) and thereby induces cell death. ETA has been used in a variety of approaches to kill tumour cells specifically. It has been used as single chain antibody fusion product to target c-erbB-2 receptors expressed in breast and ovarian cancer cells (8).

In 2000, Yerushalmi et al (9) intratumorally injected plasmids constitutively expressing a deletion mutant of ETA and reported attenuated tumour growth in immunodefficient mice. Azemar et al (10) used recombinant toxins consisting of the variable domains of the heavy and light chains of monoclonal antibodies (MAbs) genetically fused to a truncated ETA resulting in the rapid regression of subcutaneously growing oral squamous cell carcinoma tumours in nude mice. In head and neck cancer it is difficult to focus on receptor-mediated cancer therapy since up to now there is no defined surface marker exclusively expressed in this type of carcinoma. For the use of toxic agents such as ETA a tightly controlled expression is desirable. For that reason constitutive expression vectors are not suitable for gene therapy. In a recent study, we tested two environmentally inducible promoter systems in head and neck cancer cell lines: the heat sensitive Hsp70 promoter and a synthetic construct using radiation-inducible enhancer elements of the Egrl gene promoter. For expression studies we used EGFP as reporter gene. Both systems were not useful for the expression of toxin genes, since background expression was high, especially for the Egr1 promoter. Wels et al (11) have tested the expression of the catalytic domain of ETA with the Hsp70 heat-shock inducible promoter and were not able to select surviving cell clones due to the background expression of ETA in the target cells. Martin et al (12) constructed retroviral vectors, in which bacterial toxin genes were placed under the control of the thyroid hormone (T3) regulated promoter of the rat myelin basic protein. In vivo the toxin genes bearing retroviruses were able to eradicate experimentally-induced brain tumours in rats. Retroviruses are stably integrated in the host genome. Therefore, the retroviral gene transfer is specifically suitable for brain tumours because rapidly growing normal cells do not occur in the adult brain.

The GeneSwitch system is a mifepristone-inducible mammalian expression system originally developed by Wang et al (2; reviewed in 14) that uses a combination of regulatory components derived from various human and yeast transcription factors. In the GeneSwitch system, a hybrid regulatory protein containing a DNA binding domain from the yeast GAL4 protein, a truncated ligand binding domain from the human progesterone receptor, and an activation domain 
from the human NF- $\mathrm{NB}$ protein is expressed. This hybrid regulatory protein binds to the synthetic steroid, mifepristone, and functions as a ligand-dependent transcription factor to induce expression of the gene of interest as well as its own expression.

The reporter gene is expressed by the pGene/V5-His inducible expression plasmid. Expression is controlled by a hybrid promoter binding the hybrid regulatory proteinmifepristone complex (for details see www.invitrogen.com).

The GeneSwitch system has been tested in a variety of gene therapeutic approaches. Abruzzese et al (4) have used the GeneSwitch system for regulated VEGF and erythropoietin expression in vitro and in vivo and described the system as effective. GeneSwitch has also been used for tissue-specific expression in Drosophila (14) as well as for the expression of growth hormone releasing factor in mice (15). Both groups described the system as working well-defined. Adenoviral gene transfer under control of the GeneSwitch system has been used in the therapy of Parkinson's disease (reviewed in 16) and Wang et al (17) were the first team using the GeneSwitch system in transgenic animals to study the functions of the transforming growth factor $\beta 1$ in epidermal growth.

In our study we used the GeneSwitch system in combination with a deletion mutant of ETA for controlled induction of cell death in the head and neck carcinoma cell line, FADU. Carcinoma cells were effectively killed after gene induction with mifepristone. Inductivity varied from transfection to transfection and between single pSwitch clones. Even under non-suppressive culture conditions (i.e. without antibiotics) inductivity was sustained over longer time periods, pleading for stable integration of regulatory elements and the target gene into the cellular genome. Background expression level was very low, compared with different inducible reporter gene assays. The GeneSwitch system in combination with toxin genes therefore seems in principle to be suitable for head and neck cancer therapy. It provides the opportunity to switch on or off genes tightly controlled. Ngan et al (18) expressed human growth hormone (hGH) with the GeneSwitch system in combination with a liver specific promoter. They showed a 5,800-33,000-fold increase of hGH in mice serum. Induction was repeatable even after three weeks. hGH reached its maximum level $12 \mathrm{~h}$ after induction and the level declined back to basic serum values within $100 \mathrm{~h}$. In our study re-induction could effectively only be performed with surviving cells, since induction killed the cells expressing ETA. Surviving cells were less inducible than the original transfectants. This is probably due to non-proper or unfavourable integration of regulatory elements or the target gene into the cellular genome.

In summary we have demonstrated that the GeneSwitch system using a deletion mutant of ETA as a target gene is a powerful system to kill transfected tumour cells precisely after induction with the steroid mifepristone at very low doses. The system is characterized by low background expression and effective induction of the target gene. Furthermore, regulatory and target DNA seemed to be stably integrated in the host genome even after longer periods without selective pressure. This system therefore, seems to be suitable for the killing of tumour cells in vitro. Further tests will prove the usability of the GeneSwitch system in vivo.

\section{Acknowledgements}

We like to thank Professor Winfried Wels, Georg-SpeyerHaus, Institute for Biomedical Research in Frankfurt/Main for kindly providing the cDNA of ETA, and Dr Piet Tas for critical reading of the manuscript.

\section{References}

1. Schmidt M, Heimberger T, Gruensfelder P and Schler G: Inducible promoters for gene therapy of head and neck cancer: an in vitro study. Eur Arch Otorhinolaryngol 261: 208-215, 2004.

2. Wang Y, O'Malley BW Jr, Tsai SY and O'Malley BW: A regulatory system for use in gene transfer. Proc Natl Acad Sci USA 91: 8180-8184, 1994.

3. Burcin MM, Schiedner G, Kochanek S, Tsai SY and O'Malley BW: Adenovirus-mediated regulable target gene expression in vivo. Proc Natl Acad Sci USA 96: 355-360, 1999.

4. Abruzzese RV, Godin D, Mehta V,Perrard JL, French M, Nelson W, Howell G, Coleman M, O'Malley BW and Nordstrom JL: Liganddependent regulation of vascular endothelial growth factor and erythropoietin expression by a plasmid-based autoinducible GeneSwitch system. Mol Ther 2: 276-287, 2000.

5. Sirin O and Park F: Regulating gene expression using self-inactivating lentiviral vectors containing the mifepristone-inducible system. Gene 323: 67-77, 2003.

6. Bertram J: MATra - Magnet Assisted Transfection: combining nanotechnology and magnetic forces to improve intracellular delivery of nucleic acids. Curr Pharm Biotechnol 7: 277-285, 2006.

7. McFarland D: Preparation of pure cell cultures by cloning. Methods Cell Sci 22: 63-66, 2000.

8. Wels W, Harwerth I-M, Mueller M, Groner B and Hynes N: Selective inhibition of tumor cell growth by a recombinant single-chain antibody-toxin specific for the erbB-2 receptor. Cancer Res 52: 6310-6317, 1992.

9. Yerushalmi N, Brinkmann U, Brinkmann E, Pai L and Pastan I: Attenuating the growth of tumors by intratumoral administration of DNA encoding Pseudomonas exotoxin via cationic liposomes. Cancer Gene Ther 7: 91-96, 2000.

10. Azemar M, Schmidt M, Arlt F, Kennel P, Brandt B, Papadimitriou A, Groner B and Wels W: Recombinant antibody toxins specific for ErbB2 and EGF receptor inhibit the in vitro growth of human head and neck cancer cells and cause rapid tumor regression in vivo. Int J Cancer 86: 269-275, 2000.

11. Wels W, Baldrich M, Chakraborty T, Gross R and Goebel W: Expression of bacterial cytotoxin genes in mammalian target cells. Mol Microbiol 6: 2651-2659, 1992.

12. Martin V, Cortes ML, de Felipe P, Farsetti A, Calcaterra NB and Izquierdo M: Cancer gene therapy by thyroid hormone-mediated expression of toxin genes. Cancer Res 60: 3218-3224, 2000.

13. Ye X, Schillinger K, Burcin MM, Tsai SY and O'Malley BW: Ligand-inducible transgene regulation for gene therapy. Methods Enzymol 346: 551-561, 2002.

14. Osterwalder T, Yoon KS, White BH and Keshishian H: A conditional tissue-specific transgene expression system using inducible GAL4. Proc Natl Acad Sci USA 98: 12596-12601, 2001.

15. Draghia-Akli R, Malone PB, Hill LA, Ellis KM, Schwartz RJ and Nordstrom JL: Enhanced animal growth via ligand-regulated GHRH myogenic-injectable vectors. FASEB J 16: 426-428, 2002.

16. Chen Q, He Y and Yang K: Gene therapy for Parkinson's disease: progress and challenges. Curr Gene Ther 5: 71-80, 2005.

17. Wang XJ, Liefer KM, Tsai S, O'Malley BW and Roop DR: Development of gene-switch transgenic mice that inducibly express transforming growth factor betal in the epidermis. Proc Natl Acad Sci USA 96: 8483-8488, 1999.

18. Ngan ES, Schillinger K, DeMayo F and Tsai SY: The mifepristone-inducible gene regulatory system in mouse models of disease and gene therapy. Semin Cell Dev Biol 13: 143-149, 2002. 